\title{
Prediction Model of Asphalt Content of Asphalt Mixture Based on Dielectric Properties
}

\author{
Yanhui Zhong $\mathbb{D}^{1}{ }^{1}$ Yilong Wang, ${ }^{1}$ Bei Zhang $\left(\mathbb{D},{ }^{1}\right.$ Xiaolong Li $\left(\mathbb{D},{ }^{1}\right.$ Songtao Li $\left(\mathbb{D},{ }^{2}\right.$ \\ Yanmei Zhong $\mathbb{D},{ }^{3}$ Meimei Hao $\mathbb{D}^{1}{ }^{1}$ and Yanlong Gao ${ }^{1}$ \\ ${ }^{1}$ College of Water Conservancy and Environment Engineering, Zhengzhou University, No. 100 Science Rd., \\ Zhengzhou 450001, China \\ ${ }^{2}$ Zhengzhou Railway Vocational \& Technical College, No. 56 Pengcheng Rd., Zhengzhou 451460, China \\ ${ }^{3}$ Henan Provincial Academy of Building Research Co., Ltd., No. 4 Fengle Rd., Zhengzhou 450053, China
}

Correspondence should be addressed to Bei Zhang; beizhang@126.com, Xiaolong Li; wennuandeshang@hotmail.com, Songtao Li; list16@126.com, and Yanmei Zhong; zhong_yanmei@163.com

Received 25 October 2020; Revised 14 November 2020; Accepted 16 November 2020; Published 4 December 2020

Academic Editor: Jiaolong Ren

Copyright (C) 2020 Yanhui Zhong et al. This is an open access article distributed under the Creative Commons Attribution License, which permits unrestricted use, distribution, and reproduction in any medium, provided the original work is properly cited.

\begin{abstract}
The rapid detection of asphalt content in asphalt mixture is of great significance to the quality evaluation of asphalt pavement. Based on the dielectric properties of an asphalt mixture, the prediction model of asphalt content is deduced theoretically using three types of dielectric models: Lichtenecker-Rother (L-R) model, Rayleigh model, and Bottcher equation. Under the condition of laboratory mixing at room temperature (about $20-25^{\circ} \mathrm{C}$ ), a dielectric test of asphalt mixture is conducted to verify the applicability of the model. The test results indicate that the dielectric constant of the asphalt mixture is inversely proportional to the asphalt content and directly proportional to the aggregate size of the mixture. Among the models, the Rayleigh model has a wide range of applications and exhibits a high accuracy, with an average relative error of only $1.86 \%$. The results provide a theoretical basis for the nondestructive testing of asphalt pavements using ground-penetrating radar.
\end{abstract}

\section{Introduction}

Asphalt pavement is widely used in highway construction owing to its low noise, high strength, and excellent smoothness $[1,2]$. With the increasing pursuit of pavement comfort, asphalt pavements have gradually become the main structural forms of high-grade pavements [3]. The early disease of asphalt pavements is related to the construction quality, as well as the mixture oil-stone ratio. If the asphalt content is significantly low, the voidage becomes significantly high. If the asphalt content is significantly high, the thermal stability of the mixture decreases, leading to the easy onset of diseases, such as bulge and rutting. Therefore, the accurate determination of asphalt content in asphalt mixtures in the construction process plays an important role in maintaining the constructive quality of asphalt pavements [4].

At present, combustion and centrifugal extraction methods are often used to detect asphalt content [5].
However, these methods have low detective efficiency and cannot meet the requirements of rapid detection of asphalt content $[6,7]$. As a novel road quality inspection method, ground-penetrating radar (GPR) has been widely used in the quality inspection of road structure layers owing to its continuous, efficient, and nondestructive testing nature [8]. Lyntton first proposed the use of a dielectric constant to calculate pavement moisture content and compactness [9]. Topp et al. used regression analysis to establish the correlation between soil dielectric constant and soil water content [10]. In the field of soil water content detection, this model provides the most widely used empirical formula. Based on the idea of soil moisture content detection, scholars worldwide began to conduct related research on the dielectric properties of the asphalt mixture. Roth used the Time Domain Reflectometry (TDR) calibration method to measure the soil volumetric water content and dielectric constant and determined the model parameters by weighted 
regression, thereby improving the dielectric model proposed by Dobson et al. [11]. Chang et al. demonstrated the existence of a good correlation among the density, voidage, and dielectric constant of asphalt mixtures [12]. Hoegh et al. established the regression relationship between the permittivity and voidage of asphalt mixtures and used GPR to detect the compactness of pavement surfaces [13]. Song used GPR to monitor the change in surface dielectric constant with the number of vehicle loads, thus providing a theoretical basis for the real-time analysis of pavement compactness [14]. Hou used spectrophotometry to determine the asphalt content in emulsified asphalt mixtures [15], and Kumbargeri used asphaltene proportion as the performance index to study the aging of asphalt mixtures [16]. In the above literature, by studying the relationship between the dielectric constant and road moisture content, compactness, and other indexes, the idea has been applied to the study of the voidage correlation in asphalt mixtures, achieving a good effect. However, in view of the relationship between the dielectric properties and the compactness, density, and asphalt content of asphalt mixtures, a composite dielectric model to determine the asphalt content has yet to be reported.

To meet the current demand for the rapid detection of asphalt content, this study explores the feasibility of detecting asphalt content through the dielectric properties of asphalt mixtures. Using the Bottcher model, the Rayleigh model, and the L-R model, the asphalt content prediction model for the asphalt mixtures is developed through theoretical derivation. In addition, the applicability of the prediction model is verified through experiments. Furthermore, an asphalt content calculation method based on the dielectric properties of the asphalt mixtures is proposed, which provides a theoretical basis for the rapid and nondestructive testing of asphalt content using GPR.

\section{Derivation of Prediction Model of Asphalt Content}

The dielectric constant is an important parameter that reflects the dielectric properties of pavement materials. Through the analysis of the dielectric constant of pavement materials, the relevant indexes of pavement performance can be obtained [17]. Dielectric properties describe the interaction between the medium and the electric field. The dielectric properties of a medium are typically described by permittivity $\mathcal{E}$, conductivity $\sigma$, and permeability $\mu$ [18]. The dielectric constant is one of the most important parameters for characterizing the dielectric properties of a medium, reflecting the ability of the medium to store charge in the electric field. The dielectric constant of a composite medium considers the vacuum dielectric constant $\varepsilon_{0}\left(8.854 \times 10^{-9} \mathrm{~F} /\right.$ $\mathrm{m})$ as the reference value, and the relative dielectric constant of the medium is used in the simulation calculation. The ratio of dielectric constant to vacuum dielectric constant is known as the relative dielectric constant of a medium [19]. The relative permittivity is calculated as follows:

$$
\varepsilon_{r}=\frac{\varepsilon}{\varepsilon_{0}}=\varepsilon_{r}^{\prime}-j \varepsilon_{r}^{\prime \prime}
$$

It can be seen from the above equation that the relative permittivity of a medium is a dimensionless complex number. $\varepsilon_{r}^{\prime}$ is the real part of the relative dielectric constant and mainly reflects the storage effect of the medium on the electromagnetic wave. $\varepsilon_{r}^{\prime}=\sigma / \omega$ is the imaginary part of the relative dielectric constant, which mainly reflects the loss characteristics of the dielectric to the electromagnetic wave [20].

The dielectric constant of multiphase composite media can be described by the dielectric constant and volume ratio of each single-phase medium, which is known as the dielectric constant model of the composite media. There exist several dielectric constant models that describe the dielectric properties of multiphase media. The classical dielectric models include the L-R model, Rayleigh model, Bottcher equation, Bruggeman-Hanai model, and Li Jianhao model [21]. Owing to the wide application ranges of the L-R model, Rayleigh model, and Bottcher equation, the prediction accuracy of the composite dielectric constant model is high. This paper deduces the proposed model based on these three models, as described as follows.

2.1. Introduction of L-R Model. The L-R model is one of the most classical dielectric models, which has been widely used in geophysical exploration and other research fields. It can be expressed as

$$
\left(\varepsilon_{m}\right)^{c}=\sum_{i=1}^{n} f_{i}\left(\varepsilon_{i}\right)^{c} .
$$

In the above equation, the parameter $c$ assumes a value between -1 and 1 . When $c$ is $1,1 / 3$, or $1 / 2$, the $L-R$ model is converted into classical dielectric models, namely, the linear model, cubic root model, and root mean square model, respectively, as defined in equations (3), (4), and (5):

$$
\begin{gathered}
\left(\varepsilon_{m}\right)=\sum_{i=1}^{n} f_{i}\left(\varepsilon_{i}\right), \\
\left(\varepsilon_{m}\right)^{(1 / 3)}=\sum_{i=1}^{n} f_{i}\left(\varepsilon_{i}\right)^{(1 / 3)}, \\
\left(\varepsilon_{m}\right)^{(1 / 2)}=\sum_{i=1}^{n} f_{i}\left(\varepsilon_{i}\right)^{(1 / 2)} .
\end{gathered}
$$

In equations (2)-(5), $\varepsilon_{m}$ denotes the dielectric constant of the medium, $f_{i}$ is the volume ratio of the component $i$ to the mixture, and $\varepsilon_{i}$ is the dielectric constant of the component $i$.

2.2. Introduction of Rayleigh Model. When the medium is a two-phase composite, the Rayleigh model can be defined as

$$
\frac{\varepsilon_{m}-1}{\varepsilon_{m}+2}=V_{1} \frac{\varepsilon_{1}-1}{\varepsilon_{1}+2}+V_{2} \frac{\varepsilon_{2}-1}{\varepsilon_{2}+2} .
$$

The model can be extended to $n$-phase media as 


$$
\frac{\varepsilon_{m}-1}{\varepsilon_{m}+2}=\sum_{i=1}^{n} v_{i} \frac{\varepsilon_{i}-1}{\varepsilon_{i}+2} .
$$

2.3. Introduction of Bottcher Equation. Equations of symmetrical two-phase composites are as follows:

$$
V_{1} \frac{\varepsilon_{1}-\varepsilon_{m}}{\varepsilon_{1}+2 \varepsilon_{m}}+V_{2} \frac{\varepsilon_{2}-\varepsilon_{m}}{\varepsilon_{2}+2 \varepsilon_{m}}=0
$$
follows:

Dielectric model applied to multiphase composites is as

$$
\sum_{i=1}^{n} v_{i} \frac{\varepsilon_{i}-\varepsilon_{m}}{\varepsilon_{i}+2 \varepsilon_{m}}=0
$$

According to the above theoretical basis, the linear model, square root model, root mean square model, Rayleigh model, and Bottcher equation are selected to determine the asphalt content of the asphalt mixture. The derivation process and results are as follows.

2.3.1. Lichtenecker-Rother Model. Based on the L-R model, the relationship between the dielectric properties and asphalt content of the asphalt mixture was studied. The asphalt mixture was a multiphase composite medium composed of single-phase media, such as aggregate, asphalt, and air [22]. The L-R model of the asphalt mixture can be expressed by equation (10):

$$
\left(\varepsilon_{m}\right)^{c}=f_{s}\left(\varepsilon_{s}\right)^{c}+f_{a s}\left(\varepsilon_{a s}\right)^{c}+f_{a}\left(\varepsilon_{a}\right)^{c} .
$$

Here, $\varepsilon_{m}$ denotes the relative dielectric constant of the asphalt mixture. $\varepsilon_{s}, \varepsilon_{a s}$, and $\varepsilon_{a}$ are the relative dielectric constants of the aggregate, asphalt, and air in the asphalt mixture, respectively. $f_{s}, f_{a s}$, and $f_{a}$ are the volume ratios of the aggregate, asphalt, and air in the asphalt mixture. In the studied asphalt mixture, assuming $f_{s}+f_{a s}+f_{a}=1$, the volume of asphalt can be determined as follows:

$$
f_{a s}=1-f_{a}-f_{s} .
$$

The volume composition expression of the derived aggregate is obtained by combining equations (10) and (11):

$$
f_{a s}=\frac{k_{m}-k_{a s}+\left(k_{a s}-1\right) f_{a}}{k_{s}-k_{a s}} .
$$

In this equation, the expressions of the correlation coefficients $k_{m}, k_{s}, k_{a s}$, and $k_{a}$ are as follows (13). The representations of the other parameters in the equation are the same as those in equation (10).

$$
k_{i}=\left(\varepsilon_{i}\right)^{c}(i=m, s, a s, a) .
$$

The dielectric constant of air is 1 , that is, $\varepsilon_{a}=1$, and the volume ratio of air in the asphalt mixture is $f_{a}$. Therefore, the equation for voidage $e$ can be deduced as follows:

$$
e=f_{a} \times 100 \% \text {. }
$$

Considering the effect of asphalt volume ratio, the asphalt content prediction models are defined as follows:

$$
P_{a}=\frac{\gamma_{a s}(1-T-e)}{\gamma_{s} \times T+\gamma_{a s}(1-T-e)} .
$$

In the above equation, $T=\left(k_{m}-k_{a s}+\left(k_{a s}-1\right) \times(e \div 100 \%)\right) /\left(k_{s}-k_{a s}\right)$.

Using the asphalt content prediction model (15), the asphalt content can be calculated based on the dielectric properties of the asphalt mixture, which are described in detail. The value of $c$ in the L-R model of the asphalt mixture needs to be determined first [23]. In addition, the aggregate, dielectric constant, and corresponding density of the asphalt mixture must be determined. Except for the parameter $c$ in the dielectric model, the remaining parameters can be measured in the laboratory. Therefore, the dielectric model of the asphalt mixture based on the L-R model is established first, and then the asphalt content is calculated according to the prediction model.

2.3.2. Rayleigh Model. The expression of the Rayleigh model of the asphalt mixture is as follows:

$$
\begin{aligned}
\frac{\varepsilon_{m}-1}{\varepsilon_{m}+2} & =V_{s} \frac{\varepsilon_{s}-1}{\varepsilon_{s}+2}+V_{a s} \frac{\varepsilon_{a s}-1}{\varepsilon_{a s}+2}+V_{a} \frac{\varepsilon_{a}-1}{\varepsilon_{a}+2}, \\
k_{i} & =\frac{\varepsilon_{i}-1}{\varepsilon_{i}+2},(i=m, s, a s, a), \\
\varepsilon_{a} & =1, V_{a} \frac{\varepsilon_{a}-1}{\varepsilon_{a}+2}=0 .
\end{aligned}
$$

The Rayleigh model can be expressed as

$$
k_{m}=V_{s} k_{s} V_{a s} k_{a s} .
$$
(17):

The volume of the asphalt can be deduced from equation

$$
V_{a s}=1-e-V_{s} \text {. }
$$

The aggregate volume can be defined as

$$
V_{s}=\frac{k_{m}+(e-1) k_{a s}}{k_{s}-k_{a s}} .
$$

Then, the equation for asphalt content is deduced as

$$
P_{a}=\frac{\rho_{a s} V_{a s}}{\rho_{a s} V_{a s}+\rho_{s} V_{s}}=\frac{\rho_{a s}\left(1-e-V_{s}\right)}{\rho_{a s}\left(1-e-V_{s}\right)+\rho_{s} V_{s}} .
$$

2.3.3. Bottcher Equation. The expression for the Bottcher equation of the asphalt mixture is

$$
\begin{aligned}
& V_{s} \frac{\varepsilon_{s}-\varepsilon_{m}}{\varepsilon_{s}+2 \varepsilon_{m}}+V_{a s} \frac{\varepsilon_{a s}-\varepsilon_{m}}{\varepsilon_{a s}+2 \varepsilon_{m}}+V_{a} \frac{\varepsilon_{a}-\varepsilon_{m}}{\varepsilon_{a}+2 \varepsilon_{m}}=0, \\
& k_{i}=\frac{\varepsilon_{i}-\varepsilon_{m}}{\varepsilon_{i}+2 \varepsilon_{m}}(i=a, a s, a) .
\end{aligned}
$$


Then, the Bottcher equation can be expressed as

$$
V_{a s} k_{a s}+V_{s} k_{s}+V_{a} k_{a}=0
$$

The asphalt volume can be expressed as

$$
V_{a s}=1-e-V_{s} \text {. }
$$

Combining equations (22) and (23), we obtain

$$
\left(1-e-V_{s}\right) k_{a s}+V_{s} k_{s}+V_{a} k_{a}=0 .
$$

The aggregate volume can be expressed as

$$
V_{s}=\frac{(1-e) k_{a s}+V_{a} k_{a}}{k_{s}-k_{a s}} .
$$

Then, the equation for asphalt content can be deduced as

$$
P_{a}=\frac{\rho_{a s}\left(1-e-V_{s}\right)}{\rho_{a s}\left(1-e-V_{s}\right)+\rho_{s} V_{s}} .
$$

\section{Test and Method}

3.1. Test Materials. Both AC-16 asphalt concrete mixture and SMA-16 asphalt mastic macadam mixture use 90\# asphalt to prepare the standard specimens, and limestone was used as the aggregate and mineral powder. The physical parameters of the two mixtures were measured according to the requirements of the code. Based on the standard sieve system (square pore sieve) of the asphalt mixture mineral aggregate gradation screening test, the mineral aggregate was divided into $k$ grades with different particle sizes. The size of each sieve hole was recorded as $d_{m, k}$, and $r_{m, k}$ was defined as the particle size of all levels of mineral particles. The mineral material grades for the test are presented in Table 1.

3.2. Test Scheme. AC-16 and SMA-16 were selected as the experimental objects. The dielectric constants of the asphalt and aggregate single-phase medium were tested, and the dielectric properties of the AC-16 asphalt concrete and SMA-16 stone matrix asphalt with different oil-stone ratio were studied experimentally. The sample preparation process is illustrated in Figure 1, and the test process is depicted in Figure 2.

(1) According to the specifications, the AC-16 asphalt concrete mixture and SMA-16 asphalt mastic macadam mixture were prepared [11], by making the rutting plate specimen compact using a compactor [24]. The oil-stone ratios of six specimens were as follows: $4.0 \%, 4.1 \%$, and $4.2 \%$ for the first group of the AC-16 asphalt concrete mixture and 5.9\%, 6.0\%, and $6.1 \%$ for the second group. For the three standard specimens of the SMA-16 asphalt macadam mixture, the oil-stone ratios used were $6.9 \%, 7.0 \%$, and $7.1 \%$. Thus, there were nine standard specimens in total.

(2) A high-temperature coaxial probe method was used to test the dielectric constant of a single-phase medium, such as asphalt and aggregate. While testing, it is necessary to ensure that the plane of the measured object is dry and in contact with the plane of the probe. Because the dielectric constant of water at $25^{\circ} \mathrm{C}$ is approximately 81 and that of air is approximately 1 , the existence of water and voids significantly affects the measurement accuracy.

(3) The dielectric constants of the asphalt mixture specimens were measured using the waveguide cavity method of the network analyzer.

\section{Test Results and Discussion}

4.1. Main Influencing Factors of Dielectric Constant of Mixture. Figure 3 depicts the graph of the dielectric constant of asphalt and limestone versus frequency. The dielectric constants of the limestone block and asphalt are less affected by frequency in the range of $1.7-2.6 \mathrm{GHz}$. The average dielectric constants of limestone and asphalt are 7.343 and 3.002, respectively.

Based on the above test flow, the test results of the dielectric constant of the asphalt mixture are presented in Table 2. Although there is a significant difference between the oil-stone ratios of the two groups of asphalt concrete specimens (AC-16-1 to AC-16-6), their dielectric constants are similar because the asphalt concrete is composed of asphalt, coarse aggregate, fine aggregate, and mineral powder. From Figure 3, it can be observed that the average dielectric constants of the asphalt, limestone block, and mixture are approximately 3, 7, and $>5$, respectively, which indicates that the main factors affecting the dielectric constant of the mixture are the coarse and fine aggregates of limestone. Moreover, when the asphalt content increases, the dielectric constant of the mixture does not change significantly.

Although the oil-stone ratio of the asphalt mastic macadam mixture is large, the overall dielectric constant is slightly greater than that of the asphalt concrete macadam. This is because the mastic macadam mixture is composed of asphalt, coarse aggregate, mineral powder, and a small amount of fine aggregate. The proportion of coarse aggregates is relatively large corresponding to the larger dielectric constant of the complete mixture. Most of the calculation methods of the dielectric model of the mixture consider the dielectric constant and the proportion of different materials that compose the mixture and determine the contribution of each single medium to the overall dielectric constant based on its volume ratio; thus, the change in the dielectric constant can be explained.

\subsection{Effect of Asphalt Content on Dielectric Constant.} Figure 4 depicts the changing trend of the dielectric constant of the mixture with the oil-stone ratio. The dielectric constants of the three groups of specimens decrease with the increase in the oil-stone ratio. In the first and second groups, which are of the same type of asphalt concrete block, the dielectric constants decrease slightly with increasing asphalt content, and the degree of decrease follows a linear trend. In 
TABLE 1: Commonly used standard sieve sizes and corresponding particle sizes of mineral aggregate.

\begin{tabular}{lcccccccccc}
\hline$k$ & 1 & 2 & 3 & 4 & 5 & 6 & 7 & 8 & 9 & 10 \\
\hline$d_{m, k}(\mathrm{~mm})$ & 0.075 & 0.15 & 0.3 & 0.6 & 1.18 & 2.36 & 4.75 & 9.5 & 13.2 & 16 \\
$r_{m, k}(\mathrm{~mm})$ & 0.038 & 0.113 & 0.225 & 0.45 & 0.89 & 1.77 & 3.56 & 7.125 & 11.35 & 14.6 \\
\hline
\end{tabular}
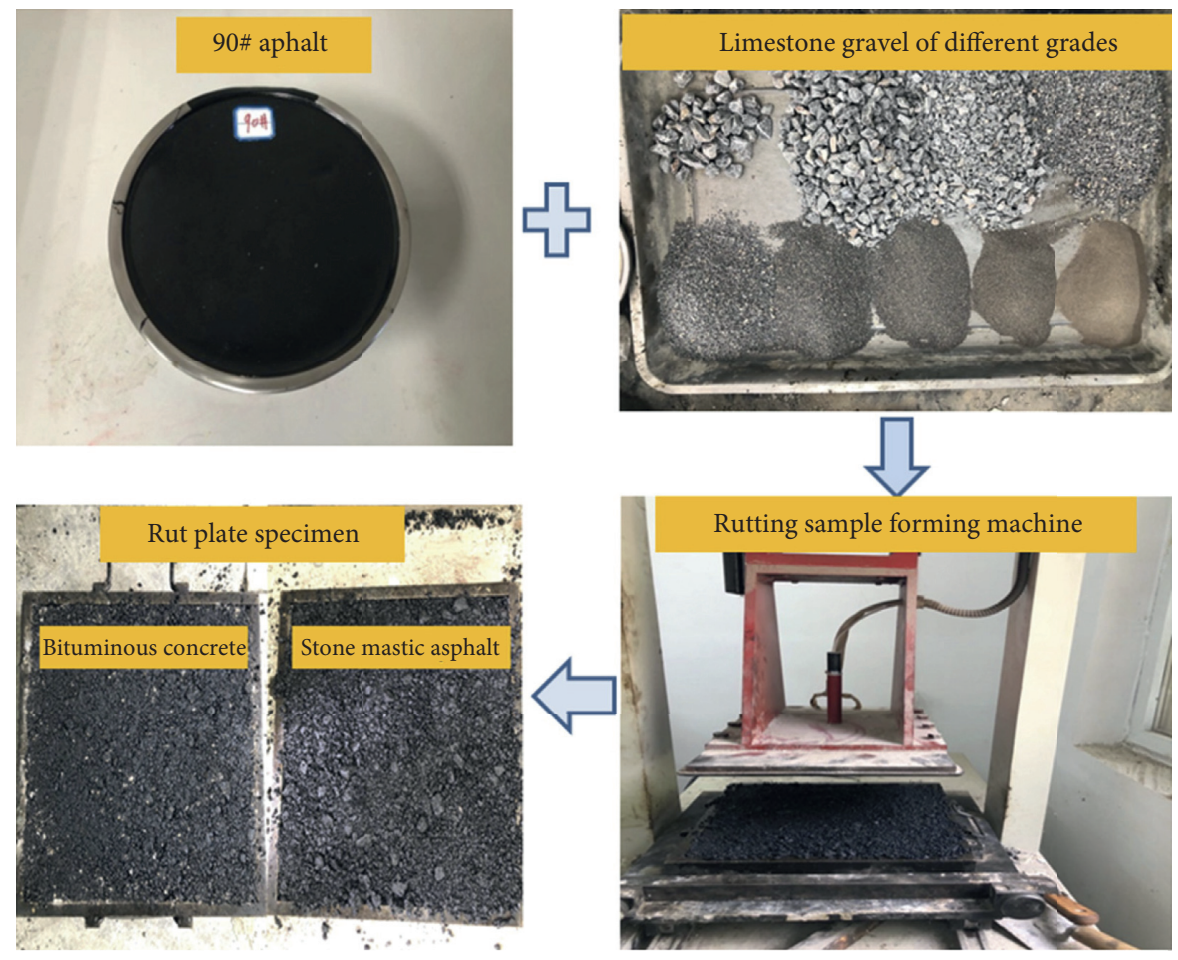

Figure 1: Production process of asphalt mixture.
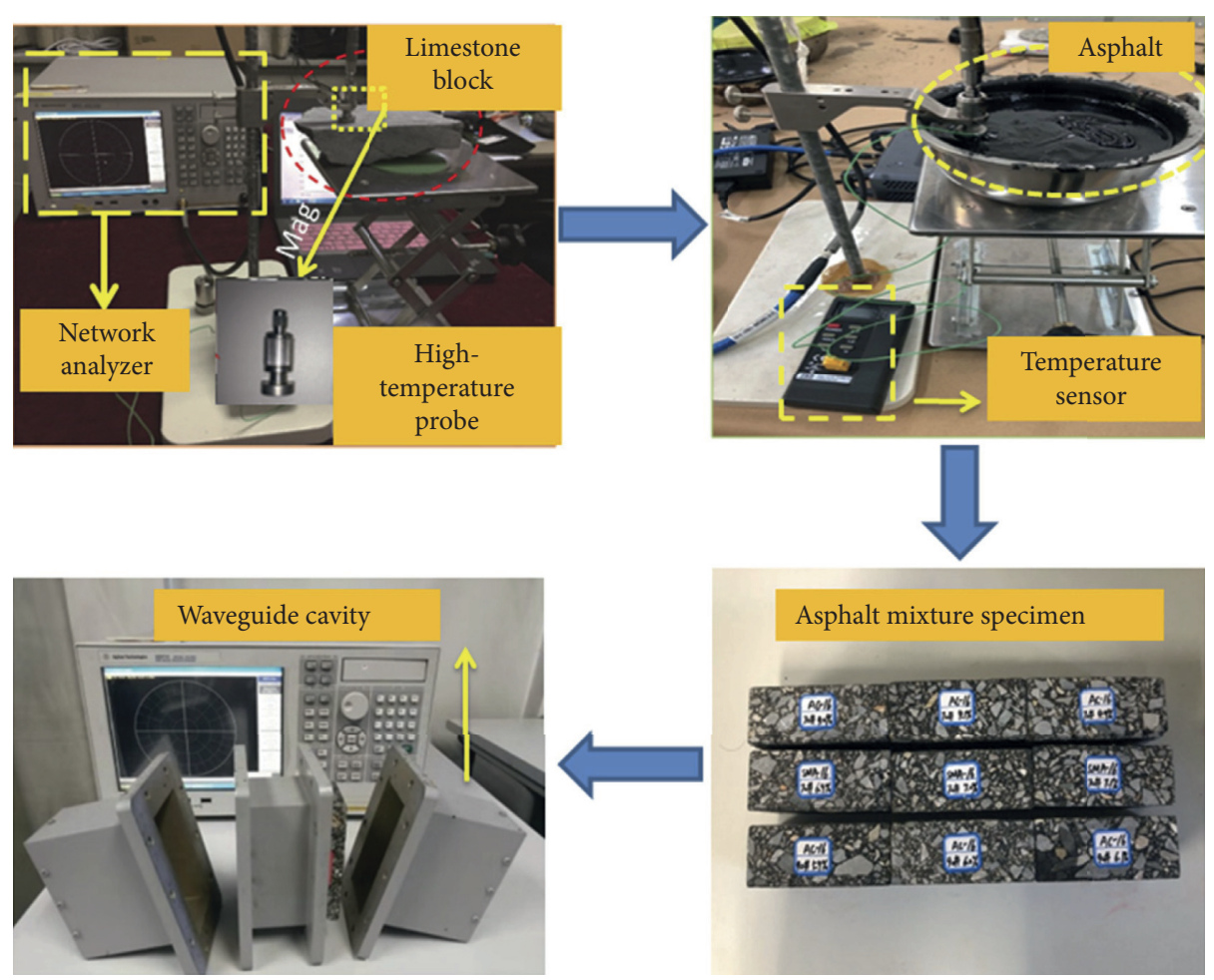

FIGURE 2: Measurement of permittivity of single medium and mixture. 

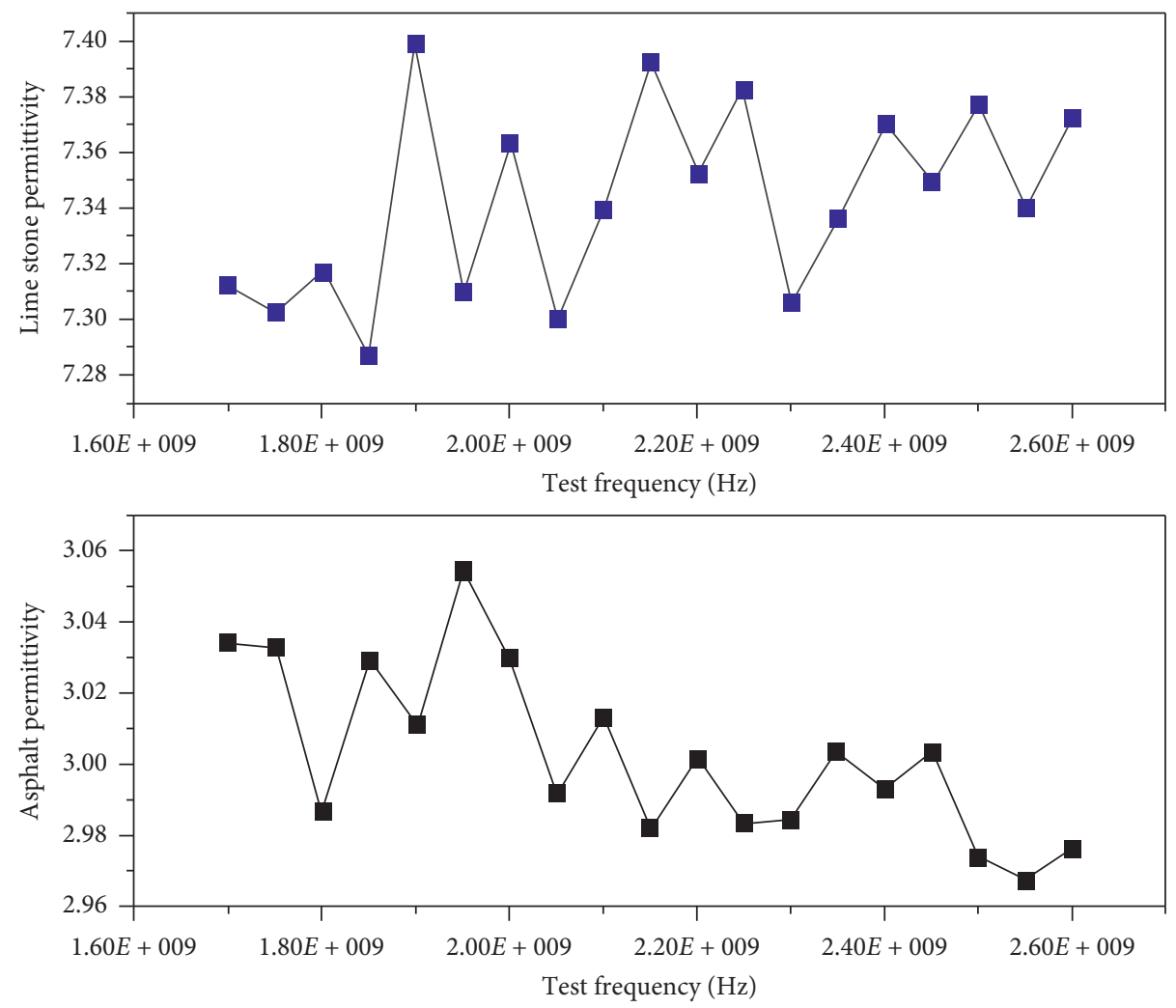

FIgURe 3: Permittivity of asphalt and limestone.

TABLE 2: Dielectric constant of asphalt mixture specimens.

\begin{tabular}{lccccccccc}
\hline Specimen number & AC-16-1 & AC-16-2 & AC-16-3 & AC-16-4 & AC-16-5 & AC-16-6 & SMA-16-1 & SMA-16-2 & SMA-16-3 \\
\hline Oil-stone ratio (\%) & 4.0 & 4.1 & 4.2 & 5.9 & 6.0 & 6.1 & 6.9 & 7.0 & 7.1 \\
Dielectric constant & 5.243 & 5.129 & 4.996 & 4.865 & 4.732 & 4.611 & 5.684 & 5.635 & 5.611 \\
\hline
\end{tabular}

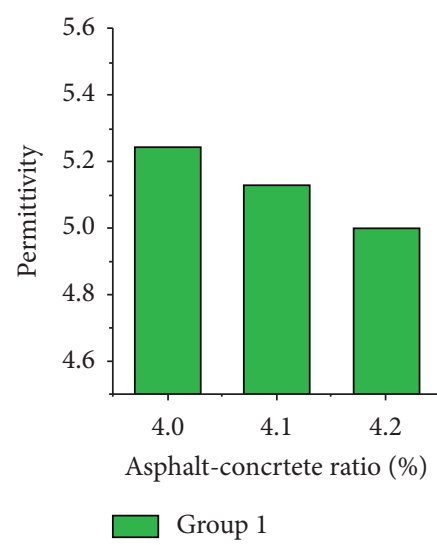

(a)

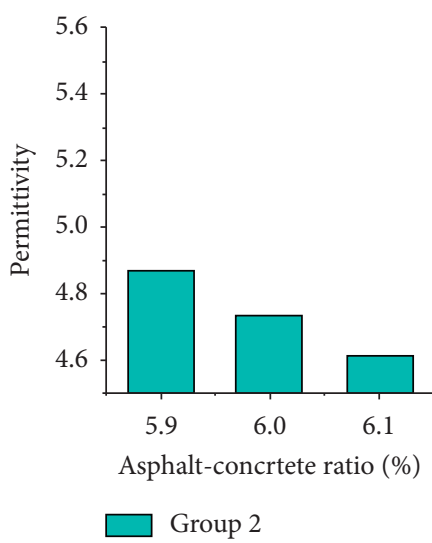

(b)

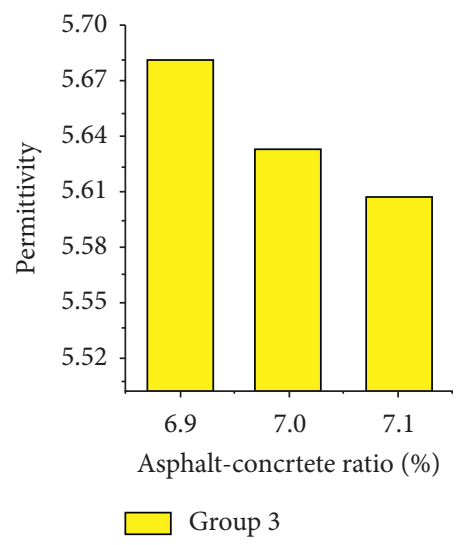

(c)

FIGURE 4: Variation of dielectric constant with the oil-stone ratio of the mixture. 
the asphalt concrete, the asphalt mortar composed of asphalt and mineral powder plays the role of bonding the coarse and fine aggregates, because the mortar requires an appropriate pigment to binder ratio to achieve the optimal bonding performance. As the asphalt content increases, the volume ratio of the coarse and fine aggregates decreases, and the filling performance of the mortar is deteriorated, resulting in an increase in voidage and a downward trend in the dielectric constant of the mixture.

Owing to the lack of a certain proportion of fine aggregates, the third group of the mastic asphalt macadam mixture contains more mineral powder, coarse aggregate, and fiber stabilizer than the other two groups, resulting in an increase in the proportion of mortar. With the increase in asphalt content, the performance of the mortar gradually improves, and the ability of the coarse aggregate to bond and fill voids is enhanced. Therefore, its dielectric constant is greater than that of the asphalt concrete block. However, according to the composite model of the dielectric constant, the main factor affecting the overall dielectric constant is the proportion of a single medium with a larger dielectric constant. As the proportion of aggregate decreases, the overall dielectric constant of the specimen still exhibits a downward trend.

\subsection{Accuracy Comparison of the Dielectric Model of Asphalt} Content. Assuming that the voidage of the mixture is known, according to the average density of the aggregate, dielectric constant, voidage of the mixture, and volume percentage of asphalt and aggregate, the data are entered into the models, such as the derived linear, square root, root mean square, Bottcher, and Rayleigh models. The calculation results are presented in Figure 5.

Figure 6 depicts the relative errors of the prediction model: the average errors of the linear, cubic root, root mean square, Rayleigh, and Bottcher models are $175.40 \%$, $85.58 \%$, $108.11 \%, 1.86 \%$, and $99.63 \%$, respectively. Composite materials are composed of two or more materials, and most of them are multiphase bodies composed of solid, liquid, and gas phases. The dielectric constant of composites is closely related to the component dielectric constant, volume ratio, geometric structure, electrochemical performance, test frequency, and temperature. Most of the dielectric constant models of composite materials cannot reflect the overall asphalt content accurately by considering the dielectric constant of the composition and the volume ratio of each phase, which is a relatively simple factor.

Although nondestructive testing technology has been developed for many years, it is still a new technology, and the research on dielectric properties of road materials is still at the initial stage, so there is no corresponding standard. Influenced by material quality, manufacturing process, mixture manufacturing process, external temperature, and other factors, the average error of dielectric property test of finished products in laboratory is generally $3 \%-5 \%$. To verify the applicability of different models to asphalt mixture, the model with error less than $10 \%$ has certain applicability.

In the L-R model, the value of parameter $c$ determines the model application range, the root mean square model is widely used in the fields of soil science and geophysics, and the cubic root model is suitable for simulating and calculating the dielectric constant of spherical and randomly distributed elliptical materials. Thus, the material type of the L-R model is different from that of the asphalt mixture. The application of the Bottcher model to twophase symmetrical composites has a good result. The Rayleigh model considers the dielectric properties of three-phase (or higher) mixed materials, and the adjustment coefficient of the parameters is close to reality. Among the test results of the five types of models, the calculation result of the Rayleigh model is closest to the measured data, while the error is the smallest, which makes it useful as a theoretical basis to conduct the practical engineering for the detection of asphalt content in asphalt pavements using GPR.

\section{Engineering Example Verification}

To verify whether the accuracy and scope of application of the model established in this study meet the requirements of engineering testing, samples were taken from the asphalt pavement of the West Fourth Ring Road in Zhengzhou, as depicted in Figure 7.

We tested the asphalt content of the samples using the combustion method and obtained the real asphalt content of the pavement surface. Using the measured dielectric constant to calculate the three volume ratios of the samples, we entered the data into the asphalt content prediction model and compared the calculated results with the measured values, as illustrated in Figure 8.

It can be observed from Figure 8 that there is a significant difference between the measured value and the calculated theoretical value of asphalt content in the asphalt pavement, and the errors of the three samples are different. The relative error and average error of the calculation are depicted in Figure 9. As can be seen from Figures 8 and 9, the relative errors of samples 1, 2, and 3 are $8.80 \%, 14.19 \%$, and $5.64 \%$, respectively, and the average error of the samples is $9.47 \%$, which is considerably higher than the accuracy of the model verified by the sample. Because the proportion of the electrical constant and volume in the asphalt mixture is minimal, using the theoretical asphalt content and dielectric constant as known conditions to calculate the three-term volume ratio of the asphalt mixture causes a large error. In addition, there is a significant difference between the asphalt mixture in the laboratory and the samples taken from the actual pavement. Thus, the main factors that must be 

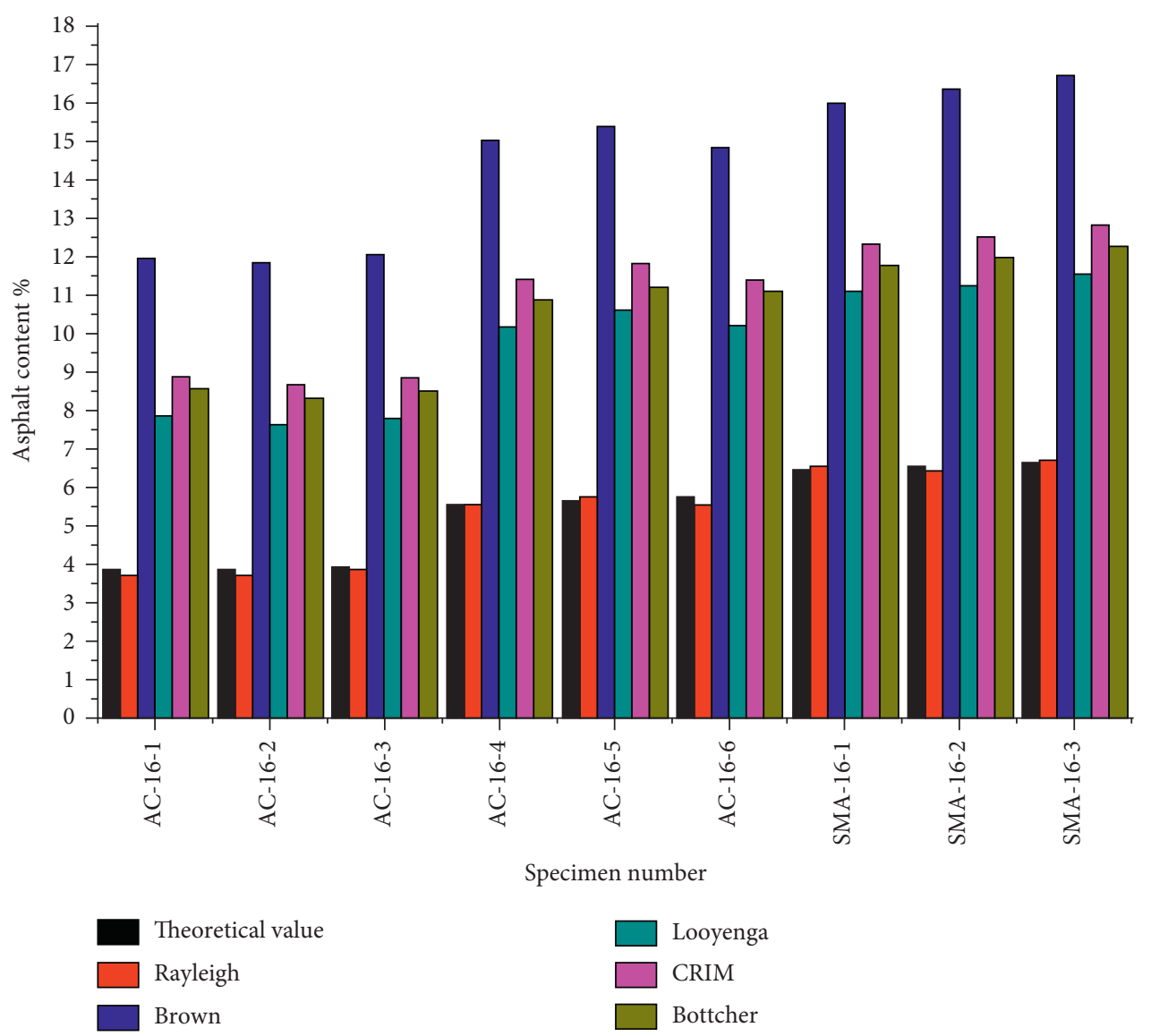

FIGURE 5: Comparison between prediction model and theoretical value.

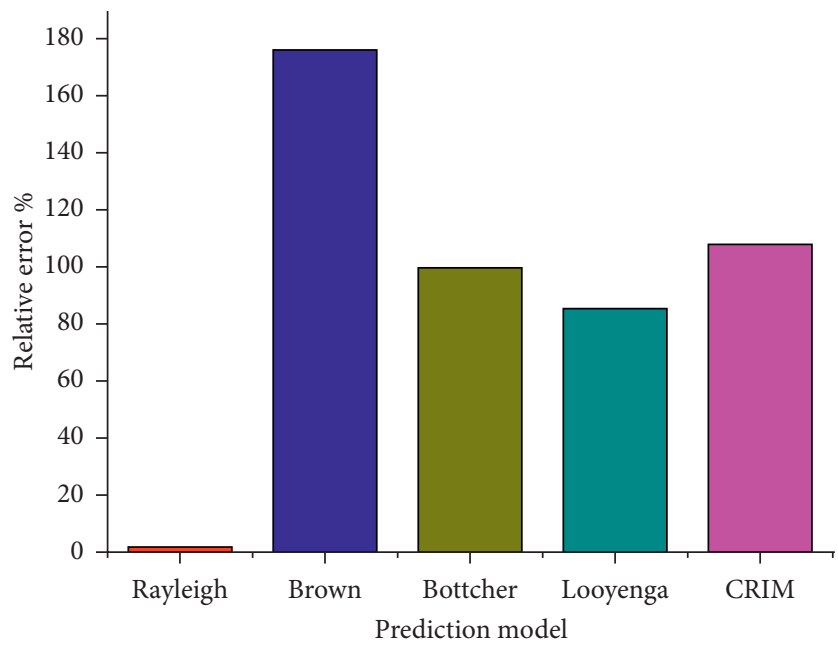

Figure 6: Relative error between prediction models. 

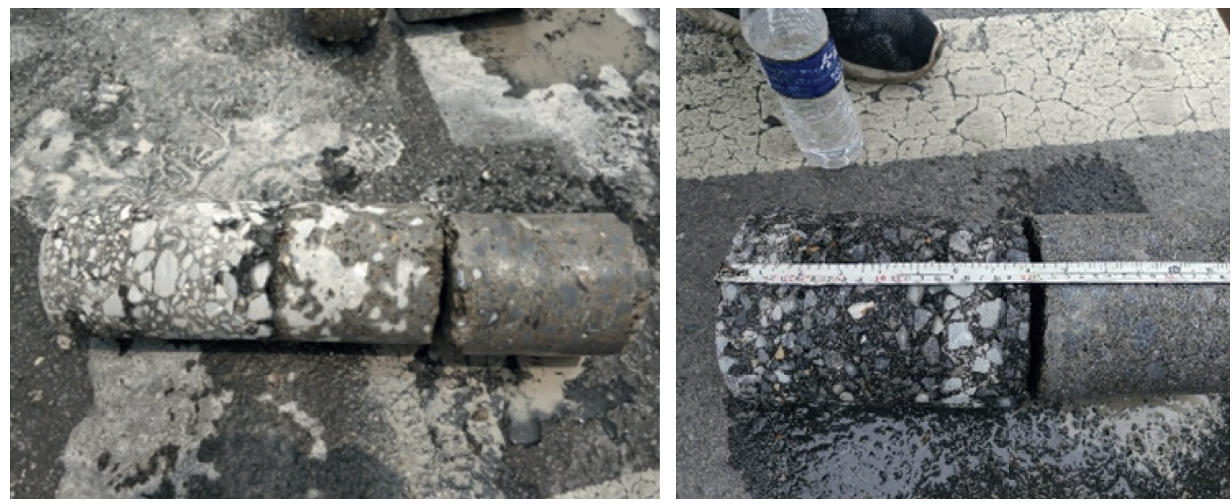

FIGURE 7: On-site sampling of asphalt pavement.

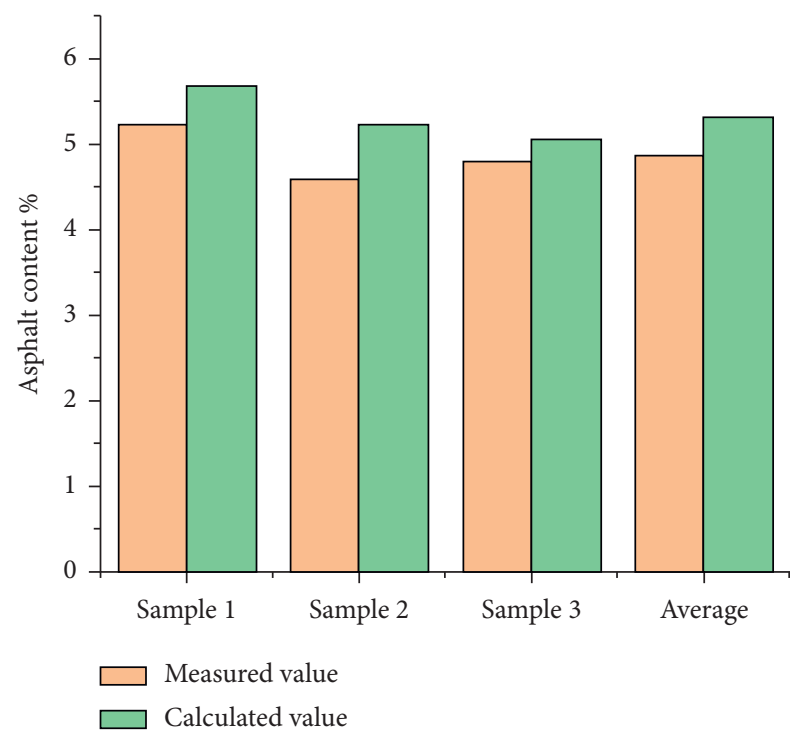

Figure 8: Comparison between the measured value and the calculated value of pavement sampling.

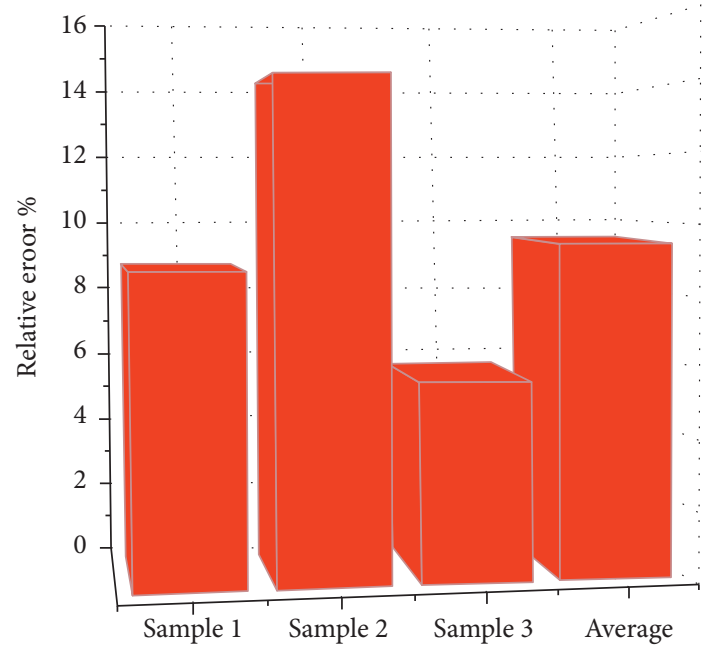

FIGURE 9: Relative error between measured and calculated values of sampling. considered are internal factors, such as compactness, voidage, ultraviolet radiation, temperature, humidity, and other external factors. In summary, the above-mentioned factors are the reason for the large error.

\section{Conclusions}

In this study, three types of dielectric models suitable for asphalt mixtures were selected from various dielectric model libraries. The correlation formula between asphalt content and dielectric constant was derived, and its accuracy was verified through experiments. The results indicate the following:

(1) In asphalt mixtures with the same gradation and mineral aggregate, as the dielectric constant of the mixture increases, the asphalt content decreases, indicating that the asphalt content is inversely proportional to the dielectric constant of the asphalt mixture.

(2) There exists a relationship between the dielectric constant of the asphalt mixture and the particle size of the aggregate. Owing to the large dielectric constant due to the large particle size of the aggregate, the dielectric constant of the mixture increases with an increase in the aggregate size of the same type of asphalt.

(3) According to the dielectric model of the composite, the proportion of the single medium in the mixture determines the dielectric constant of the mixture, and the single medium with a large dielectric constant is the main factor affecting the dielectric constant of the mixture. This is supported by the fact that the proportion of coarse aggregates in the asphalt mastic mixture is relatively large, and the dielectric constant of the asphalt mastic mixture is greater than that of the asphalt concrete of the same grade.

(4) Among the established models, the calculation results of the Rayleigh model for the asphalt concrete block and asphalt mastic macadam mixture are closer to the test value, exhibiting higher accuracy. 
The minimum error is $0.18 \%$, the maximum error is $3.38 \%$, and the average error is $1.86 \%$. The Rayleigh model exhibits the best effect in terms of both the scope of application and accuracy rate. As an important theoretical basis for road GPR detection of the asphalt content in asphalt pavements, this study lays the foundation for follow-up work.

\section{Data Availability}

The data used to support the findings of this study are included within the article.

\section{Conflicts of Interest}

The authors declare that they have no conflicts of interest.

\section{Acknowledgments}

The authors acknowledge the support received from the National Key Research and Development Plan (Grant no. 2018YFB1600200), the National Natural Science Foundation of China (Grants nos. 51878624 and 51878622), Major Scientific and Technological Special Project in Henan (Grant no. 181100310400), the Program for Innovative Research Team (in Science and Technology) at the University of Henan Province (Grant no. 18IRTSTHN007), the Program for Science and Technology Innovation Talents in Universities of Henan Province (Grant no. 19HASTIT041), Natural Science Foundation of Henan Province (Grant no.202300410746), and Key Research Projects of Higher Education in Henan Province (Grant no. 18A580001).

\section{References}

[1] S. Sonny Kim, J. J. Yang, and R. Austin Etheridge, "Effects of mix design variables on flexibility index of asphalt concrete mixtures," International Journal of Pavement Engineering, vol. 21, p. 1275, 2020.

[2] J. E. Edeh, U. Terry, and S. A. Okpe, "The use of cement treated reclaimed asphalt pavement-quarry waste blends as highway material," International Journal of Pavement Engineering, vol. 21, p. 1191, 2020.

[3] K. Hoegh, T. Steiner, E. Zegeye Teshale, and S. Dai, "Minnesota department of transportation case studies for coreless asphalt pavement compaction assessment," Transportation Research Record: Journal of the Transportation Research Board, vol. 2674, no. 2, p. 291, 2020.

[4] H. N. Yu, X. Zhu, and G. P. Qian, "Evaluation of phosphorus slag (PS) content and particle size on the performance modification effect of asphalt," Construction and Building Materials, vol. 256, Article ID 119334, 2020.

[5] A. Baltrusaitis, A. Vaitkus, and J. Smirnovs, "Asphalt layer density and air voids content: GPR and laboratory testing data reliance," Baltic Journal of Road and Bridge Engineering, vol. 15, p. 93, 2020.

[6] H. Liu and M. Sato, "In situ measurement of pavement thickness and dielectric permittivity by GPR using an antenna array," NDT and E International, vol. 64, p. 65, 2014.

[7] L. Zhen, I. L. Al-Qadi, and S. Lahouar, "Development and validation for in situ asphalt mixture density prediction models," NDT and E International, vol. 44, p. 369, 2011.
[8] T. Liu, X.-N. Zhang, Z. Li, and Z.-Q. Chen, "Research on the homogeneity of asphalt pavement quality using X-ray computed tomography (CT) and fractal theory," Construction and Building Materials, vol. 68, p. 587, 2014.

[9] E. J. Jaselskis, J. Grigas, and A. Brilingas, "Dielectric properties of asphalt pavement," Journal of Materials in Civil Engineering, vol. 15, no. 5, p. 427, 2003.

[10] G. C. Topp, J. L. Davis, and A. P. Annan, "Electromagnetic determination of soil water content: measurements in coaxial transmission lines," Water Resources Research, vol. 16, no. 3, p. $574,1980$.

[11] K. Roth, R. Schulin, H. Fluhler, and W. Attinger, "Calibration of time domain reflectometry for water content measurement using a composite dielectricity approach," Water Resources Research, vol. 26, no. 10, p. 2267, 1990.

[12] C.-M. Chang, J.-S. Chen, and T.-B. Wu, "Dielectric modeling of asphalt mixtures and relationship with density," Journal of Transportation Engineering, vol. 137, no. 2, p. 104, 2011.

[13] E. Z. Teshale, K. Hoegh, and S. D. Dai, "Ground penetrating radar sensitivity to marginal changes in asphalt mixture composition," Journal of Testing and Evaluation, vol. 48, no. 3, Article ID 20190486, 2020.

[14] A. L. Song and J. Gagnon, "Monitoring hot mix asphalt pavement density changes using ground penetrating radar at the FAA's national airport pavement test facility," in Proceedings of the 15th International Conference on Ground Penetrating Radar, p. 919, Brussels, Belgium, July 2014.

[15] X. Hou, F. Xiao, R. Guo, Q. Xiang, T. Wang, and J. Wang, "Application of spectrophotometry on detecting asphalt content of emulsified asphalt," Journal of Cleaner Production, vol. 215, p. 626, 2019.

[16] Y. S. Kumbargeri and K. P. Biligiri, "A novel approach to understanding asphalt binder aging behavior using asphaltene proportion as a performance indicator," Journal of Testing and Evaluation, vol. 44, p. 439, 2016.

[17] J. Mao, L. Sun, J. Lv, and Y. Tan, "Piezoelectric and dielectric behavior of 0-3 asphalt-based composites with carbon black," Ceramics International, vol. 42, no. 14, p. 16132, 2016.

[18] J. Hu, P. K. R. Vennapusa, D. J. White, and I. Beresnev, "Pavement thickness and stabilised foundation layer assessment using ground-coupled GPR," Nondestructive Testing and Evaluation, vol. 31, no. 3, p. 267, 2016.

[19] K. Hoegh, S. Dai, T. Steiner, and L. Khazanovich, "Enhanced model for continuous dielectric-based asphalt compaction evaluation," Transportation Research Record: Journal of the Transportation Research Board, vol. 2672, no. 26, p. 144, 2018.

[20] C. Plati and A. Loizos, "Using ground-penetrating radar for assessing the structural needs of asphalt pavements," Nondestructive Testing and Evaluation, vol. 27, no. 3, p. 273, 2012.

[21] P. Shangguan, I. L. Al-Qadi, and S. Lahouar, "Pattern recognition algorithms for density estimation of asphalt pavement during compaction: a simulation study," Journal of Applied Geophysics, vol. 107, p. 8, 2014.

[22] D.-H. Chen, F. Hong, W. Zhou, and P. Ying, "Estimating the hotmix asphalt air voids from ground penetrating radar," NDT and E International, vol. 68, p. 120, 2014.

[23] S. Wang, S. Zhao, and I. L. Al-Qadi, "Continuous real-time monitoring of flexible pavement layer density and thickness using ground penetrating radar," NDT and E International, vol. 100, p. 48, 2018.

[24] A. Ahmed, K. Emad, and M. Eyad, "Method for predicting the laboratory compaction behavior of asphalt mixtures," Journal of Materials in Civil Engineering, vol. 27, 2015. 